\title{
Mesh-based numerical implementation of the localized boundary-domain integral equation method to a variable-coefficient Neumann problem
}

\author{
S. E. Mikhailov and I. S. Nakhova \\ Division of Mathematics, School of Computing and Mathematical Sciences, \\ Glasgow Caledonian University, Glasgow, G4 OBA, UK
}

\begin{abstract}
An implementation of the Localized Boundary-Domain Integral Equation (LBDIE) method to numerical solution of the Neumann boundary-value problem for a second order linear elliptic PDE with variable coefficient is discussed. The LBDIE method uses a specially constructed localized parametrix (Levi function) to reduce the BVP to a localized boundary-domain integral equation. After employing a mesh-based discretization, the integral equation is reduced to a sparse system of linear algebraic equations that is solved numerically. Since the Neumann BVP is not unconditionally and uniquely solvable, neither is the LBDIE. Numerical implementation of the finite-dimensional perturbation approach that reduces the integral equation to an unconditionally and uniquely solvable equation, is also discussed.
\end{abstract}

Keywords: Linear Partial Differential Equation, Integral Equation, Localized parametrix, Cut-off function, Finite-dimensional Perturbation, Sparse Matrix

\section{Introduction}

The boundary element method, based on the boundary integral equations, is a well established technique for solution of many problems with constant coefficients. However, the finite element method appears to be much more effective for variable coefficient problems.

In order to reduce a Boundary Value Problem (BVP) for a Partial Differential Equation (PDE) to a boundary integral equation useful for numerical implementation, a fundamental solution of the PDE must be available in an analytical form and/or cheaply computable. Such fundamental solutions are well-known for many constantcoefficient PDEs. Particularly, the BVPs for the classical Laplace equation can be reduced to a boundary integral equation and then solved by the boundary element method, see e.g. [2].

Unfortunately, such a fundamental solution is not available in the general case of the partial differential operators with coefficients varying throughout the domain. PDEs with variable coefficients arise frequently in applications involving inhomogeneous media (e.g., functionally graded materials, [1]). An option for problems with variable coefficients is to use a parametrix (Levi function) instead of a fundamental solution for the reduction of the BVP to a boundary-domain integral equation (BDIE), 
see e.g. [3] and references therein. However, discretization of the BDIE leads then to a dense system of linear algebraic equations, and hence prevents the use of economical methods that are available for sparse systems.

To avoid this difficulty, it was suggested in [4] to employ a specially constructed localized parametrix, reducing BVPs with variable coefficients to a Localized BoundaryDomain Integral Equation (LBDIE). After a mesh-based or mesh-less discretization, this results in a sparse system of linear algebraic equations, that can be solved by well-known efficient methods.

Another type of LBDIE for BVP of elasticity with variable coefficients was described in [6], where the Green function for a corresponding homogeneous material on a sphere was employed as a parametrix. The mesh-less method based on the MLS approximation, c.f. $[7,8]$, and the element-wise polynomial approximation were used for numerical implementations in [6]. A localized boundary-domain integro-differential equation, which is equivalent to use of the piece-wise constant cut-off function, c.f. [4], was used in [9] for numerical solution for the BVP of heat transfer for an inhomogeneous material in conjunction with the piece-wise polynomial approximation of the solution.

In the present paper, we outline implementation of the mesh-based discretization of the LBDIE formulated in [4] and associated with the Neumann BVP for a linear scalar variable-coefficient equation. Numerical solutions for some test problems with variable coefficients are discussed, extending results of [5]. This illustrates applicability of the discrete algorithms presented in [4] for solution of the BDIEs localized with smooth cut-off functions.

\section{Reduction of the Neumann BVP to LBDIE}

Let us consider the following second-order linear elliptic PDE in a two-dimensional bounded domain $\Omega$,

$$
[L u](x):=\frac{\partial}{\partial x_{i}}\left[a(x) \frac{\partial}{\partial x_{i}} u(x)\right]=f(x), \quad x \in \Omega,
$$

subject to the Neumann boundary condition

$$
[T u](x):=a(x) \frac{\partial u(x)}{\partial n(x)}=\bar{t}(x), \quad x \in \partial \Omega,
$$

where $u(x)$ is the unknown function; $f(x), \bar{t}(x)$ and $a(x)>$ const $>0$ are prescribed functions; $n(x)$ is the outward normal unit vector to the boundary $\partial \Omega$ of the domain $\Omega ; x=\left(x_{1}, x_{2}\right)$; and summation over the repeated indices is implied from 1 to 2 .

The BVP (1)-(2) appears particularly in modeling stationary heat transfer, elastostatics, electrostatics and diffusion problems for functionally graded materials, as well as in fluid mechanics of porous media.

To ensure compatibility of boundary condition (2) with equation (1), we assume that the functions $\bar{t}(x)$ and $f(x)$ satisfy the compatibility condition, c.f. [10],

$$
\int_{\partial \Omega} \bar{t}(x) d \Gamma(x)-\int_{\Omega} f(x) d \Omega(x)=0 .
$$


For partial differential operators with variable coefficients, a parametrix can be defined as a function $P(x, y)$ satisfying the equation

$$
[L P(\cdot, y)](x)=\delta(x-y)+R(x, y),
$$

where $\delta(x-y)$ is the Dirac delta-function, $y$ is a source point, and the remainder $R(x, y)$ as a function of point $x \in \Omega$, may have at $x=y$ a weak singularity at most.

In the $2 \mathrm{D}$ case, the fundamental solution of the operator with 'frozen coefficients' corresponding to the operator $L$ defined in (1), can be taken as a parametrix, i.e.,

$$
P(x, y)=\frac{1}{2 \pi a(y)} \ln |x-y|,
$$

where $|x-y|=\sqrt{\left(x_{1}-y_{1}\right)^{2}+\left(x_{2}-y_{2}\right)^{2}}, \ln |x-y|=\log _{e}|x-y|$.

After substituting (4) in (3), the remainder $R(x, y)$ can be calculated as

$$
R(x, y)=\frac{x_{i}-y_{i}}{2 \pi a(y)|x-y|^{2}} \frac{\partial a(x)}{\partial x_{i}} .
$$

The localized parametrix is defined as

$$
P_{\omega}(x, y)=\chi(x, y) P(x, y),
$$

where $\chi(x, y)$ is a sufficiently smooth cut-off function, defined such that $\chi(y, y)=1$ and $\chi(x, y)=0$ if $x \notin \bar{\omega}(y) ; \omega(y)$ is an open localization domain, whose closure $\bar{\omega}(y)$ contains the point $y$. Some examples of $\bar{\omega}(y)$ are shown on Fig. 1.

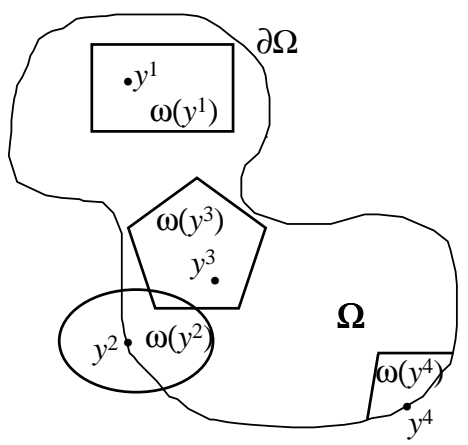

Figure 1: A domain $\Omega$ with possible localization domains $\omega\left(y^{i}\right)$.

Further, we have

$$
\begin{aligned}
{\left[L P_{\omega}(\cdot, y)\right](x)=} & {[L\{\chi(\cdot, y) P(\cdot, y)\}](x)=} \\
& {[L P(\cdot, y)](x)+[L\{(1-\chi(\cdot, y)) P(\cdot, y)\}](x)=\delta(x, y)+R_{\omega}(x, y) . }
\end{aligned}
$$

Hence,

$$
R_{\omega}(x, y)=R(x, y)+[L\{(1-\chi(\cdot, y)) P(\cdot, y)\}](x) .
$$

The second Green identity over the intersection of $\omega(y)$ with $\Omega$, for the operator $L$ from (1), unknown solution $u$ and an arbitrary test function $v$, has the form,

$$
\int_{\omega(y) \cap \Omega}[u(x)[L v](x)-v(x)[L u](x)] d \Omega(x)=\int_{\partial[\omega(y) \cap \Omega]}[u(x)[T v](x)-v(x)[T u](x)] d \Gamma(x) .
$$


Substituting the localized parametrix $P_{\omega}(x, y)$ for the test function $v(x)$ in the identity, taking the usual limits in the singular point $x=y$, see e.g. [3, Sec. I.9], and taking into account boundary condition (2), we arrive at the direct LBDIE, c.f. [4],

$$
\begin{aligned}
c(y) u(y) & -\int_{\bar{\omega}(y) \cap \partial \Omega} u(x)\left[T P_{\omega}(\cdot, y)\right](x) d \Gamma(x)-\int_{\partial \omega(y) \cap \Omega} u(x)\left[T P_{\omega}(\cdot, y)\right](x) d \Gamma(x) \\
& +\int_{\partial \omega(y) \cap \Omega} P_{\omega}(x, y)[T u](x) d \Gamma(x)+\int_{\omega(y) \cap \Omega} R_{\omega}(x, y) u(x) d \Omega(x) \\
=- & \int_{\bar{\omega}(y) \cap \partial \Omega} P_{\omega}(x, y) \bar{t}(x) d \Gamma(x)+\int_{\omega(y) \cap \Omega} f(x) P_{\omega}(x, y) d \Omega(x), \quad y \in \bar{\Omega}
\end{aligned}
$$

where

$$
c(y)=\left\{\begin{array}{cl}
1, & \text { for } y \in \Omega, \\
\frac{\alpha(y)}{2 \pi}, & \text { for } y \in \partial \Omega,
\end{array}\right.
$$

and $\alpha(y)$ is the interior space angle at a point $y$ of the boundary $\partial \Omega$; particularly, $c=1 / 2$ at a smooth boundary point.

Note that each point $y$ generally possesses its own localization domain $\omega(y)$ in LBDIE (7); by other words, the localization domain moves with $y$. The second and third integrals on the left-hand side of LBDIE (7) disappear if the cut-off function $\chi$ is chosen such that

$$
\chi(x, y)=\frac{\partial \chi(x, y)}{\partial n(x)}=0 \quad \text { at } \quad x \in \partial \omega(y) \cap \Omega,
$$

and consequently

$$
P_{\omega}(x, y)=\frac{\partial P_{\omega}(x, y)}{\partial n(x)}=0 \quad \text { at } \quad x \in \partial \omega(y) \cap \Omega .
$$

The localized boundary-domain integral equations like (7) consist a new nontraditional class of equations not covered by the classical theory of the boundarydomain integral equations presented e.g. in [3]. They have some connections with the domain decomposition technique (c.f. comments in [4]) but essentially differ from it by the fact that the localization domain moves with the field point $y$; that is, each point $y$ generally possesses its own localization domain $\omega(y)$.

\section{Finite-dimensional perturbation}

The Neumann BVP (1)-(2) is not unconditionally and uniquely solvable, see e.g. [10], and neither is the integral equation. Consequently, the system of linear algebraic equations, which will be a discrete analogue of the LBDIE (7), is either singular or ill-conditioned. For such types of systems, direct methods, particularly the Gauss elimination, can give unstable solutions, i.e. small changes in the coefficients of the system's matrix or in the right-hand side, which can be caused by computer round-off errors, may produce large changes in the solution. Iterative methods for such systems may converge very slowly, if at all. The situation can be improved following the 
approach described in [11]. One could check that $u(x)=$ const is a solution of the LBDIE (7) with the zero right-hand side. Let us assume there are no other solutions of the homogeneous equation, and only one compatibility condition is to be imposed on inhomogeneous LBDIE (7). Then one can perturb LBDIE (7) by adding the operator

$$
[\stackrel{\circ}{K} u](x):=\frac{1}{|\partial \Omega|} \int_{\partial \Omega} u(x) d \Gamma(x),
$$

where $|\partial \Omega|$ denotes the length of the boundary $\partial \Omega$, to the left-hand side of the LBDIE (7). The perturbed LBDIE can be written as

$$
\begin{gathered}
c(y) u(y)-\int_{\bar{\omega}(y) \cap \partial \Omega} u(x)\left[T P_{\omega}(\cdot, y)\right](x) d \Gamma(x)-\int_{\partial \omega(y) \cap \Omega} u(x)\left[T P_{\omega}(\cdot, y)\right](x) d \Gamma(x) \\
+\int_{\partial \omega(y) \cap \Omega} P_{\omega}(x, y) T_{x} u(x) d \Gamma(x)+\int_{\omega(y) \cap \Omega} R_{\omega}(x, y) u(x) d \Omega(x)+\frac{1}{|\partial \Omega|} \int_{\partial \Omega} u(x) d \Gamma(x) \\
=-\int_{\bar{\omega}(y) \cap \partial \Omega} P_{\omega}(x, y) \bar{t}(x) d \Gamma(x)+\int_{\omega(y) \cap \Omega} f(x) P_{\omega}(x, y) d \Omega(x)
\end{gathered}
$$

This equation is uniquely and unconditionally solvable, and its solution is a particular solution of equation (7) such that

$$
\frac{1}{|\partial \Omega|} \int_{\partial \Omega} u(x) d \Gamma(x)=0
$$

c.f. [11].

Discretization of the perturbed LBDIE should lead to a system of linear algebraic equations with a well-conditioned matrix.

\section{Discretization of the LBDIEs}

Let us discretize the domain $\Omega$ into a mesh of triangular elements $\bar{e}_{k}, e_{k} \cap e_{m}=\emptyset$, $k \neq m$, with nodes $x^{i}, i=1, \ldots J$, at the vertices of triangles, and choose localization domains $\bar{\omega}\left(x^{i}\right)$ consisting of the elements adjacent to the node $x^{i}$, i.e., $\bar{\omega}\left(x^{i}\right)=\bigcup_{x^{i} \in \bar{e}_{k}} \bar{e}_{k}$, see Fig. 2. This means that each localization domain will shrink with the mesh refinement in our localization approach, unlike in traditional domain decomposition methods.

Let $s_{k}$ be the side of the element $\bar{e}_{k}$, constituting $\bar{\omega}\left(x^{i}\right)$, which does not pass through $x^{i}$. For such localization domains $\omega\left(x^{i}\right)$, the smooth cut-off function can be taken as

$$
\chi\left(x, x^{i}\right)=\left\{\begin{array}{ccc}
\prod_{\substack{k=1 \\
x^{i} \notin \bar{s}_{k}}}^{p} \frac{\rho_{k}^{2}(x)}{\rho_{k}^{2}\left(x^{i}\right)} & \text { if } \quad x \in \bar{\omega}\left(x^{i}\right), \\
0 & \text { if } \quad x \notin \bar{\omega}\left(x^{i}\right),
\end{array}\right.
$$

where $p$ is a number of triangles $\bar{e}_{k}$ in $\omega\left(x^{i}\right), \rho_{k}(x), \rho_{k}\left(x^{i}\right)$ are the distances from points $x$ and $x^{i}$ to the side $s_{k}$ of $\bar{e}_{k}$. For the nodes $x^{i}$ on the global boundary $\partial \Omega$, like point $x^{2}$ in Fig. 2, formula (11) implies that $\chi\left(x, x^{i}\right) \neq 0$ at $x$ on the intersection 


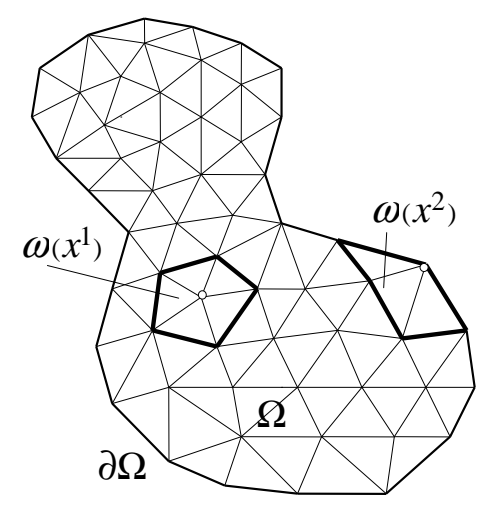

Figure 2: Mesh-based discretization and localization domains $\omega\left(x^{i}\right)$.

$\bar{\omega}\left(x^{i}\right) \cap \partial \Omega$. Obviously, the cut-off function $\chi\left(x, x^{i}\right)$, given by (11), and the localized parametrix $P_{\omega}\left(x, x^{i}\right)$, given by (6), (4), satisfy conditions (8), (9).

To obtain the system of linear algebraic equations from the LBDIE (7) or from its perturbed counterpart (10) by the collocation method, we apply this equation at the nodes $x^{i}, i=1, \ldots J$, and substitute an interpolation of $u(x)$ of the form

$$
u(x) \approx \sum_{x^{j} \in \bar{\omega}\left(x^{i}\right)} u\left(x^{j}\right) \Phi_{j}(x), \quad \Phi_{j}(x)= \begin{cases}\phi_{k j}(x) & \text { if } x, x^{j} \in \bar{e}_{k} \subset \bar{\omega}\left(x^{i}\right), \\ 0 & \text { otherwise, }\end{cases}
$$

where $\phi_{k j}(x)$ are the shape functions localized on an element $\bar{e}_{k}$, associated with the node $x^{j}$. For the triangle elements, $\phi_{k j}(x)$ can evidently be chosen as linear functions equal to 1 at $x_{j} \in \bar{e}_{k}$, and 0 at other vertices of the triangle $e_{k} ; \Phi_{j}(x)$ is then a piece-wise linear function.

The system of linear algebraic equations obtained after the discretization of LBDIE (7) can be written as

$$
c\left(x^{i}\right) u\left(x^{i}\right)+\sum_{x^{j} \in \bar{\omega}\left(x^{i}\right)} K_{i j} u\left(x^{j}\right)=\mathcal{F}\left(x^{i}\right), \quad i=1, \ldots, J,
$$

where

$$
\begin{gathered}
K_{i j}=-\int_{\bar{\omega}\left(x^{i}\right) \cap \partial \Omega} \Phi_{j}(x)\left[T P_{\omega}\left(\cdot, x^{i}\right)\right](x) d \Gamma(x)+\int_{\omega\left(x^{i}\right) \cap \Omega} \Phi_{j}(x) R_{\omega}\left(x, x^{i}\right) d \Omega(x), \\
\mathcal{F}\left(x^{i}\right)=-\int_{\bar{\omega}\left(x^{i}\right) \cap \partial \Omega} P_{\omega}\left(x, x^{i}\right) \bar{t}(x) d \Gamma(x)+\int_{\omega\left(x^{i}\right) \cap \Omega} f(x) P_{\omega}\left(x, x^{i}\right) d \Omega(x) .
\end{gathered}
$$

Since $K_{i j}=0$ at $x^{j} \notin \bar{\omega}\left(x^{i}\right)$, the number of non-zero entries equals the number of nodes in $\bar{\omega}\left(x^{i}\right)$ (the typical number is not more than 8 ), i.e. the matrix is sparse with an effective band width around 8 .

Discretizing perturbed LBDIE (10), we arrive at the system

$$
c\left(x^{i}\right) u\left(x^{i}\right)+\sum_{x^{j} \in \bar{\omega}\left(x^{i}\right)} K_{i j} u\left(x^{j}\right)+\sum_{x^{j} \in \partial \Omega} \stackrel{\circ}{K_{j}} u\left(x^{j}\right)=\mathcal{F}\left(x^{i}\right), \quad i=1, \ldots, J,
$$

where $\stackrel{\circ}{K}=\frac{1}{|\partial \Omega|} \int_{\partial \Omega} \Phi_{j}(x) d \Gamma(x)$. The last system involves the sparse matrix $K_{i j}$ and the column matrix $\stackrel{\circ}{K}$. 


\section{Numerical results}

The algorithm was implemented in a computer code with the localized parametrix $P_{\omega}(x, y)$ given by (6), (4), and the cut-off function $\chi(x, y)$ given by (11). The integrals in formulae (13) and (14) have a weak singularity of the order of $O(\ln |x-y|)$. To calculate the boundary integrals we used a logarithmically weighted Gaussian quadrature, see e.g. [12]. For the domain integrals, a degenerate transformation of variable, see e.g. [13, Sec. 11.3], has been implemented, mapping the triangles into squares and eliminating the weak singularity.

For comparison, both systems (12) and (15) were solved, giving the same results. To solve system (12), we used the procedure LFSXG of the numerical library IMSL that exploit the sparsity pattern. For the solution of perturbed system (15), IMSL routines LFCRG and LFIRG have been adopted. The condition numbers for the systems were estimated by using the routine LFCRG. In our numerical experiments, the condition numbers for the non-perturbed system were of the order of $10^{6}-10^{8}$, while for the perturbed system were of the order of $10-10^{3}$.

To verify the convergence of the method, we applied the LBDIE method to some test problems on square and circular domains, for which an exact analytical solution, $u_{\text {exact }}$, is available. The relative error was calculated as

$$
\varepsilon(J)=\frac{\max _{1 \leq j \leq J}\left|u_{\text {approx }}\left(x^{j}\right)-u_{\text {exact }}\left(x^{j}\right)\right|}{\max _{1 \leq j \leq J}\left|u_{\text {exact }}\left(x^{j}\right)\right|} .
$$

Since the exact solution is not unique, the approximate solution was taken as $u_{\text {approx }}\left(x^{j}\right)=$ $\widetilde{u}_{\text {approx }}\left(x^{j}\right)-\widetilde{u}_{\text {approx }}\left(x^{1}\right)+u_{\text {exact }}\left(x^{1}\right), \quad j=1, \ldots, J$, where $\widetilde{u}_{\text {approx }}$ is the numerical solution.

The following numerical tests were performed.

Test 1. Square domain $\bar{\Omega}=\left\{\left(x_{1}, x_{2}\right):-1 \leq x_{1}, x_{2} \leq 1\right\}$, where $a(x)=x_{1} x_{2}+2$, $f(x)=0$ for $x \in \bar{\Omega}$ and $\bar{t}(x)=2\left(x_{1} x_{2}+2\right)\left(x_{1} n_{1}(x)-x_{2} n_{2}(x)\right)$ for $x \in \partial \Omega$. An exact solution for this problem is $u_{\text {exact }}(x)=x_{1}^{2}-x_{2}^{2}, \quad x \in \bar{\Omega}$. 


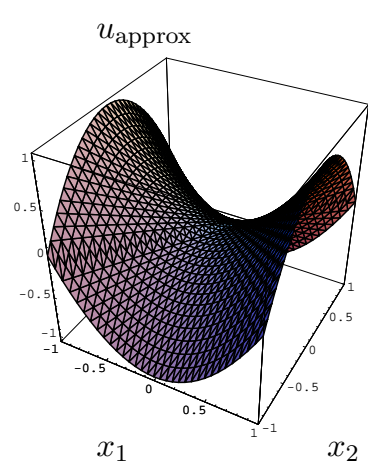

(a)

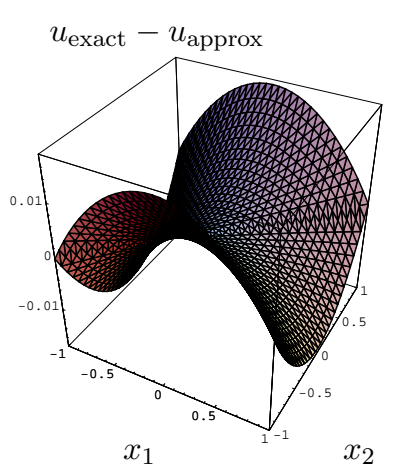

(b)

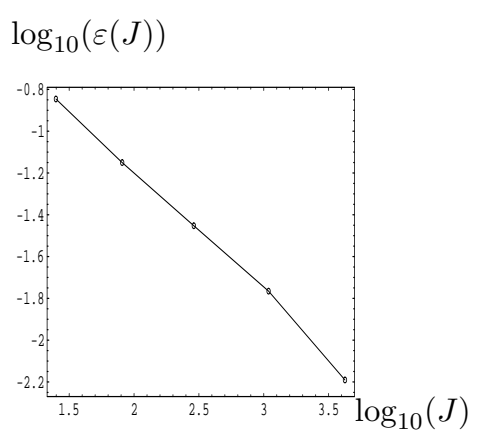

(c)

Figure 3: Results for test 1: (a) approximate solution $u_{\text {approx }}(x)$, (b) difference between approximate and exact solutions, when $J=1089, \varepsilon(1089) \approx 1.7 \%$; (c) relative error versus number of nodes.

Test 2. Square domain $\bar{\Omega}=\left\{\left(x_{1}, x_{2}\right): 1 \leq x_{1}, x_{2} \leq 2\right\}$, where $a(x)=x_{1}^{2}+x_{2}^{2}$, $f(x)=8\left(x_{1}^{2}+x_{2}^{2}\right)$ for $x \in \bar{\Omega}$ and $\bar{t}(x)=2\left(x_{1}^{2}+x_{2}^{2}\right)\left(x_{1} n_{1}(x)+x_{2} n_{2}(x)\right)$ for $x \in \partial \Omega$. An exact solution for this problem is $u_{\text {exact }}(x)=x_{1}^{2}+x_{2}^{2}, \quad x \in \bar{\Omega}$.

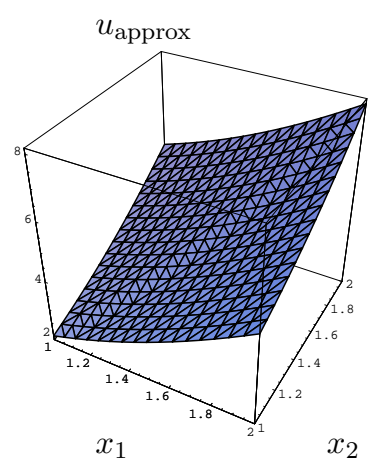

(a)

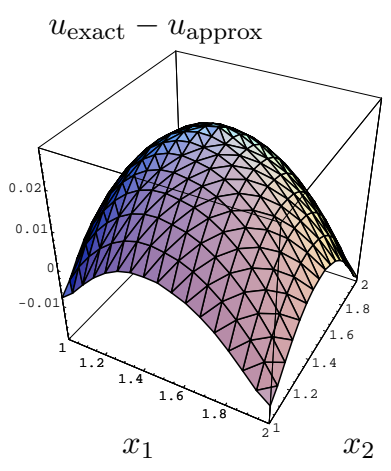

(b)

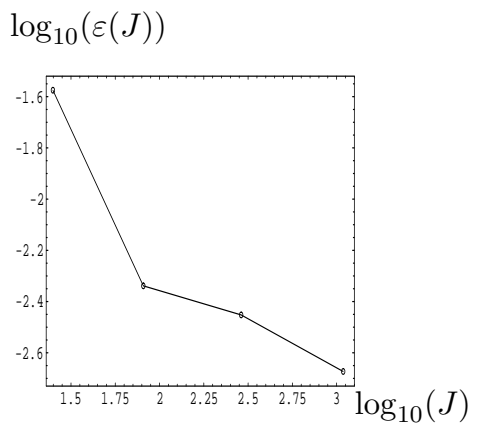

(c)

Figure 4: Results for test 2: (a) approximate solution $u_{\text {approx }}(x),(\mathbf{b})$ difference between approximate and exact solutions, when $J=289, \varepsilon(289) \approx 0.35 \%$; (c) relative error versus number of nodes.

Test 3. Circular domain $\bar{\Omega}=\left\{\left(x_{1}, x_{2}\right):\left(x_{1}-1,5\right)^{2}+\left(x_{2}-1,5\right)^{2} \leq 0.25\right\}$, where $a(x)=x_{1}^{2}+x_{2}^{2}, f(x)=8\left(x_{1}^{2}+x_{2}^{2}\right)$ for $x \in \bar{\Omega}$ and $\bar{t}(x)=2\left(x_{1}^{2}+x_{2}^{2}\right)\left(x_{1} n_{1}(x)+x_{2} n_{2}(x)\right)$ for $x \in \partial \Omega$. An exact solution for this problem is $u_{\text {exact }}(x)=x_{1}^{2}+x_{2}^{2}, \quad x \in \bar{\Omega}$.

Fig.5 and Fig.6 show the results of Test 3 for the circular domain with the nondiscretization-consistent and the discretization-consistent choices for the coefficient $c\left(x^{i}\right), x^{i} \in \partial \Omega$, respectively. In the former case $\alpha\left(x^{i}\right)=\frac{1}{2}, x^{i} \in \partial \Omega$, as should be for the smooth circle boundary. In the latter case, the boundary nodes $x^{i}$ are considered as vertices with the angles $\alpha\left(x^{i}\right)$ of the polygon approximating the circle 
in the implemented mesh-based discretization, and $c\left(x^{i}\right)=\frac{\alpha\left(x^{i}\right)}{2 \pi}$. The discretizationconsistent choice gives higher accuracy for the circular domain.

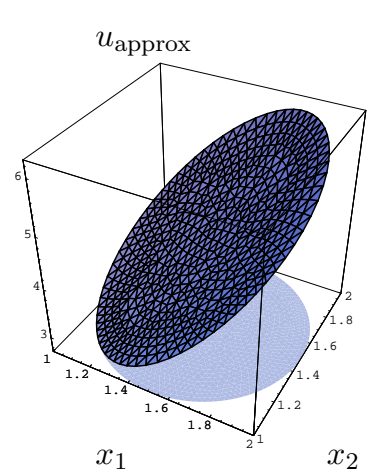

(a)

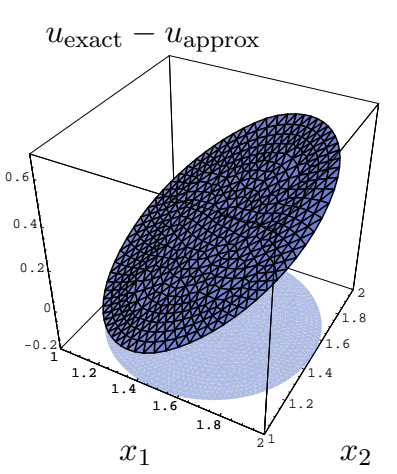

(b)

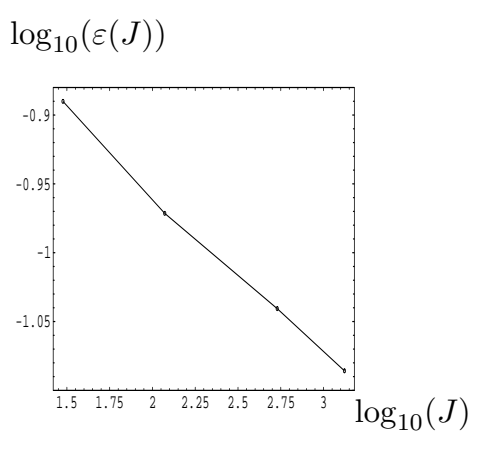

(c)

Figure 5: Results for test 3 with $c\left(x^{i}\right)=\frac{1}{2}$ on $\partial \Omega$ : (a) approximate solution $u_{\text {approx }}(x)$, (b) difference between approximate and exact solutions when $J=536, \varepsilon(536) \approx 9.1 \%$.

(c) relative error versus number of nodes

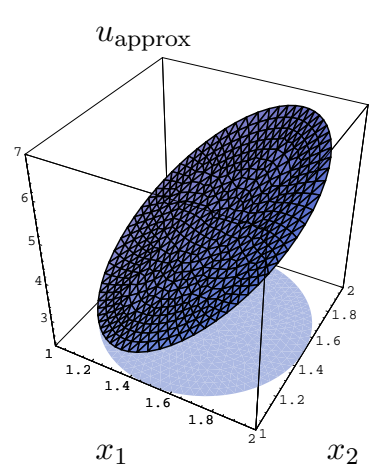

(a)

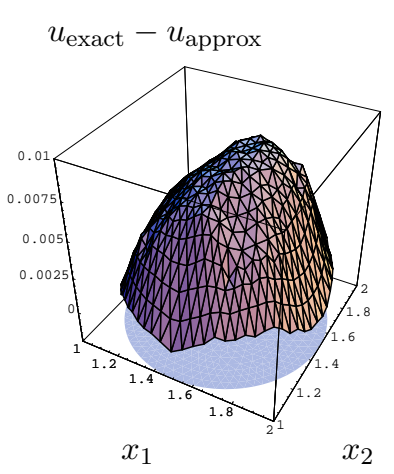

(b)

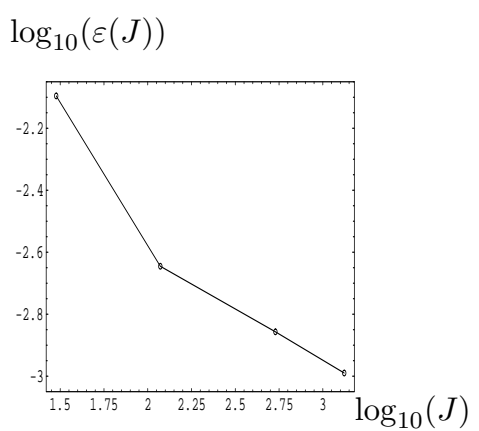

(c)

Figure 6: Results for test 3 with $c\left(x^{i}\right)=\frac{\alpha\left(x^{i}\right)}{2 \pi}$ on $\partial \Omega$ : (a) approximate solution $u_{\text {approx }}(x),(\mathbf{b})$ difference between approximate and exact solutions when $J=536$, $\varepsilon(536) \approx 0.14 \%$. (c) relative error versus number of nodes

\section{Concluding remarks}

Localization of the parametrix by multiplication with a locally supported cut-off function reduces a BVP to a localized boundary-domain integral equation. This ends up after discretization in a sparse system of linear algebraic equations, which makes the method competitive with the Finite Element method.

The numerical experiments show that after the mesh-based discretization the LBDIE method with a smooth cut-off function leads to a convergent algorithm and, consequently, is applicable as a numerical method for solution of BVPs with variable 
coefficients. Further theoretical and numerical analysis of the convergence rate and error estimates would be beneficial for optimal choice of the cut-off functions and especially their smoothness.

The finite-dimensional perturbation allows to reduce the integral equation of the Neumann problem to an unconditionally and uniquely solvable integral equation. This will be especially beneficial for constructing iterative numerical methods for solving corresponding algebraic systems without preconditioning.

\section{References}

1. S. Suresh and A. Mortensen, Fundamentals of Functionally Graded Materials. London: Institute of Materials (1998) 165 pp.

2. C.A. Brebbia, J.C.F. Telles and L.C. Wrobel, Boundary Element Techniques. Berlin: Springer (1984) 464 pp.

3. C. Miranda, Partial Differential Equations of Elliptic Type. Berlin - Heidelberg - New York: Springer (1970) 370pp.

4. S.E. Mikhailov, Localized boundary-domain integral formulations for problems with variable coefficients. Engineering Analysis with Boundary Elements 26 (2002) 681-690.

5. S.E. Mikhailov and I.S. Nakhova, Numerical solution of a Neumann problem with variable coefficients by the localized boundary-domain integral equation method. In: S. Amini (ed.), Fourth UK Conference on Boundary Integral Methods, ISBN 0-902896-40-7, Salford: Salford University (2003) pp.175-184.

6. J. Sladek, V. Sladek and S. N. Atluri, Local boundary integral equation (LBIE) method for solving problems of elasticity with nonhomogeneous material properties. Computational Mechanics 24 (2000) 456-462.

7. T. Zhu, J.-D. Zhang and S.N. Atluri, A local boundary integral equation (LBIE) method in computational mechanics, and a meshless discretization approach. Computational Mechanics 21 (1998) 223-235.

8. T. Zhu, J.-D. Zhang and S.N. Atluri, A meshless numerical method based on the local boundary integral equation (LBIE) to solve linear and non-linear boundary value problems. Engineering Analysis with Boundary Elements 23 (1999) 375-389.

9. V. Sladek and J. Sladek, A new formulation for solution of boundary value problems using domain-type approximations and local integral equations. Electronic Journal of Boundary Elements 1 (2003) 132-153.

10. Mikhlin, S. G. Mathematical physics, an advanced course. Amsterdam, London: North-Holland Publishing (1970), 561 pp.

11. S.E. Mikhailov, Finite-dimensional perturbations of linear operators and some applications to boundary integral equations, Engineering Analysis with Boundary Elements 23 (1999) 805-813.

12. A.H. Stroud and D. Secrest, Gaussian quadrature formulas. Englewood Cliffs: Prentice-Hall (1966) 374pp. 
13. L.C. Wrobel, The Boundary Element Method, Vol. 1, Applications in ThermoFluids and Acoustics. Chichester: Wiley (2002) 451pp. 